\title{
Optimizing the radiologist work environment: Actionable tips to improve workplace satisfaction, efficiency, and minimize burnout
}

\author{
Minu Agarwal ${ }^{1,2} \cdot$ Christian B. van der Pol ${ }^{1,2}\left(\right.$ Michael N. Patlas $^{2} \cdot$ Amar Udare $^{1,2} \cdot$ Andrew D. Chung $^{3,4}$. \\ Julian Rubino ${ }^{4}$
}

Received: 24 May 2021 / Accepted: 5 July 2021 / Published online: 16 July 2021

(C) Italian Society of Medical Radiology 2021

Medical imaging has become increasingly widespread and available. Radiologists are often required to perform more with less as imaging examinations increase in volume and complexity, which is often not matched by proportionate increase in staffing [1]. This can have a negative effect on the wellbeing of radiologists and patients, and ultimately lead to radiologist burnout. Radiologists have higher burnout rates than most other specialists with workplace stress a contributing factor [2]. Chronic stress can lead to emotional exhaustion, depersonalization, and a sense of reduced personal accomplishment, particularly in the setting of limited resources and poor engagement. Professional burnout in healthcare can negatively impact professionalism, productivity, healthcare costs, and importantly patient care [3]. To

Christian B. van der Pol

vanderpolc@hhsc.ca

Minu Agarwal

agarwalmi@hhsc.ca

Michael N. Patlas

patlas@hhsc.ca

Amar Udare

udare@hhsc.ca

Andrew D. Chung

andrew.chung@queensu.ca

Julian Rubino

jrubino@qmed.ca

1 Department of Diagnostic Imaging, Department of Radiology, Juravinski Hospital and Cancer Centre, Hamilton Health Sciences, 711 Concession Street, Hamilton, ON L8V 1C3, Canada

2 Department of Radiology, McMaster University, Hamilton Health Sciences, 711 Concession Street, Hamilton, ON L8V 1C3, Canada

3 Department of Radiology, Kingston Health Sciences Centre, 76 Stuart Street, Kingston, ON K7L2V7, Canada

4 Queen's University, Kingston, Canada mitigate radiologist burnout, we have identified several contributing factors and provide departmental and individual evidence-based strategies to address these by improving workplace satisfaction and radiologist efficiency.

\section{Diagnostic imaging department tips}

Diagnostic imaging departments should embrace technological solutions to ensure harmonious workflow. Striving for seamless integration of the digital work environment including the picture archiving and communication system (PACS), radiological information system (RIS), examination protocol entry platform, electronic medical records (EMR), voice recognition software, and instant messaging systems has been a successful informatics-based solution [4]. This involves automating processes at each step to save radiologist time and frustration by minimizing the number of inputs required from the radiologist to summon the medical record, import clinical data from the record into the report, and to convey important findings to referring physicians. While artificial intelligence programs are being developed to help with interpretative tasks, additional non-radiologist personnel can be employed to help with time-intensive tasks, sometimes referred to as "radiology preprocessors." Preliminary results on interpretative assistants trained to annotate and measure specific findings show $37 \%$ reduction in radiologists' average dictation time [5]. Auto-populating specific imaging findings such as measurements in ultrasound reports with the assistance of special software can also save valuable time [4]. Technologists can also be trained to assign examination protocols. For radiologists supervising scans, PACSintegrated instant messaging is effective for communication between radiologists and technologists, and can reduce the number of disturbances requiring the radiologist's attention outside of the workstation [4]. 
Extracting from the Eudaimonia architectural concepts tested at The Children's Hospital of Philadelphia, different sections within the department may be created where the level of distraction aligns with the task being performed. A less accessible section can be turned into a "distraction-free" area to allow deep focussed work, while the on-call service can occur in an area allowing for moderate to considerable distraction [6].

Achieving successful communication with referring physicians can be convoluted and time-consuming for radiologists. Radiology coordinators can facilitate radiologistreferrer communication which can improve radiologist productivity. These personnel can act as the first point of contact within the diagnostic imaging department for incoming phone calls, faxes and emails, can connect referrers to the appropriate radiologists, and can aid communication between radiologists and technologists. We recommend implementation of an automated alert notification system for critical test results and accessible real-time updated contact information for the most responsible physician. Utilizing email or messaging applications for important but non-urgent communication with receipt acknowledgement to establish closed-loop communication is recommended.

\section{Radiologist technology tips}

Radiologists can leverage technology to their advantage. Optimizing multi-step display protocols and dictation software macros with picklists can all be significant time savers. Similarly, programmable and ergonomic multi-button computer mice (often designed for computer gaming) can avoid the need to switch back and forth between the keyboard and mouse when interpreting examinations by assigning keyboard shortcuts to mouse buttons (via mouse software) [7]. This may seem daunting but can increase productivity in the long run.

Social media has become a major force in the lives of many radiologists and provides more value than simply "socializing." Many use social media to distribute educational material, to discuss complex cases, to highlight key events in the specialty, and to discuss research (mentions of a study on social media provide a measure of study impact, e.g. Altmetric Attention Score) [8]. Social media can, however, be a distraction, and turning off notifications for social media applications for part or all of the workday, or simply keeping phone settings on "do not disturb," can help alleviate disruptive alerts. To manage incoming emails, batching and automated responses can also help reduce distractions. Author Tim Ferris in his book The 4-h workweek suggests an autoresponder template [9]: "I am currently checking and responding to email twice daily at__A.M. and__P.M. If you require urgent assistance, please contact me via phone at (extension/phone number). Thank you for understanding this move to help you serve better."

\section{Radiologist workflow tips}

With seemingly ever-increasing demands on diagnostic imaging, tasks including assigning examination protocols, answering pages and phone calls, presenting at multidisciplinary conferences, and in-person consults are frequent and often interrupt workflow which can lead to diagnostic errors for both the radiologist involved and others sharing the workspace. Such interruptions may often disengage one completely from the primary interpretative task at hand. Assigning examination protocols routinely between examination interpretation, rather than allowing the list of examinations needing protocols to accumulate, minimizes the risk of being interrupted to assign a pending protocol. Standing protocols can be implemented at the department level for specific indications. For instance, unenhanced CT KUB for nephrolithiasis and unenhanced head CT for trauma [4].

Using shared drives to collect, maintain, and distribute the latest evidence-based guidelines amongst colleagues can save time and improve consistency within a group. Many cloud-based services are available that can be used for this purpose.

\section{Radiologist physical and emotional wellbeing}

Optimizing ergonomics is important for radiologists to avoid workplace-related injury. Height-adjustable desks, adjustable chairs with armrests and lumbar support, and optimization of ambient light and noise can all improve the work environment [10]. Wellness at work is important for a productive and pleasant work environment. Investing in sessions with wellness and ergonomic experts is something all departments should consider.

Time should be allocated to tasks that bring a sense of purpose to an individual and ameliorate isolation. These can include teaching, interprofessional collaborations, shared decision making, and opportunities for career advancement. High levels of engagement tend to occur in situations where employees have a sense of autonomy, competency, and have meaningful social interactions.

\section{Conclusion}

Like all medical specialities, Radiology has unique challenges. Both individual-level and department-level strategies are necessary to optimize the work environment. Adapting 
technology-based solutions, hiring support staff, and optimizing existing resources can help increase a department's output while maintaining radiologist efficiency and promoting wellbeing. There is a need to integrate self-care into the workplace. Positive social interactions with other physicians, trainees, and support staff can nurture psychosocial engagement.

Authors' contributions All authors contributed to this manuscript: Drs. Agarwal, van der Pol and Patlas conceived the idea and its relevance in the current times; Drs. Agarwal, Udare, Chung and Rubiano did the literature review. All authors contributed to drafting and revisions of the submitted manuscript.

Funding No funding was received to assist with the preparation of this manuscript.

\section{Declarations}

Conflict of interest Dr. Patlas received an editorial honorarium from Springer. Additional authors have no conflicts of interest to declare.

Ethical approval Not applicable.

Consent for publication The manuscript does not contain clinical studies or patient data.

\section{References}

1. Bhargavan M, Kaye AH, Forman HP, Sunshine JH (2009) Workload of radiologists in United States in 2006-2007 and trends
Since 1991-1992. Radiology 252:458-467. https://doi.org/10. 1148/radiol.2522081895

2. Medscape National Physician Burnout \& Suicide Report 2020: The Generational Divide. In: Medscape. https://www.medscape. com/slideshow/2020-lifestyle-burnout-6012460. Accessed $30 \mathrm{Apr}$ 2021

3. West CP, Dyrbye LN, Shanafelt TD (2018) Physician burnout: contributors, consequences and solutions. J Intern Med 283:516529. https://doi.org/10.1111/joim. 12752

4. Doshi AM, Moore WH, Kim DC et al (2018) Informatics solutions for driving an effective and efficient radiology practice. Radiographics 38:1810-1822. https://doi.org/10.1148/rg.2018180037

5. Do HM, Spear LG, Nikpanah M et al (2020) Augmented radiologist workflow improves report value and saves time: a potential model for implementation of artificial intelligence. Acad Radiol 27:96-105. https://doi.org/10.1016/j.acra.2019.09.014

6. Larsen EP, Hailu T, Sheldon L et al (2021) Optimizing radiology reading room design: the Eudaimonia radiology machine. J Am Coll Radiol 18:108-120. https://doi.org/10.1016/j.jacr.2020.09. 041

7. Denton K, Haider I, Hill J et al (2018) Of mice and roentgen: radiologist satisfaction with a non-conventional 13-Button MouseOne Institution's experience. J Digit Imaging 31:776-782. https:// doi.org/10.1007/s10278-018-0094-7

8. Warren HR, Raison N, Dasgupta P (2017) The rise of altmetrics. JAMA 317:131-132. https://doi.org/10.1001/jama.2016.18346

9. Ferriss T (2009) The 4-hour Workweek: Escape 9-5, Live Anywhere, and Join the New Rich. Crown Publishers

10. Harisinghani MG, Blake MA, Saksena M et al (2004) Importance and effects of altered workplace ergonomics in modern radiology suites. Radiographics 24:615-627. https://doi.org/10.1148/ rg.242035089

Publisher's Note Springer Nature remains neutral with regard to jurisdictional claims in published maps and institutional affiliations. 